\title{
Efeitos da sinvastatina na hipertrofia lipídica da medula óssea induzida pelo uso de corticóides em ratos ${ }^{*}$
}

\author{
Effects of simvastatin in steroid-induced \\ bone marrow lipidic hypertrophy in rats
}

\author{
Liszt Palmeira de Oliveira ${ }^{1}$, LUiz Otávio Sampaio Penteado², \\ Jorge José de Carvalho ${ }^{3}$, Karlos Celso de Mesouita ${ }^{4}$
}

\section{RESUMO}

Objetivos: Avaliar o efeito do uso da sinvastatina (SV) sobre a área da medula ocupada por adipócitos (AMOA) na cabeça femoral de ratos com hipertrofia lipídica induzida pelo uso de hidrocortisona (HC). Métodos: Foram utilizados 40 ratos (Rattus norvegicus albinus), machos, da linhagem Wistar, distribuídos em cinco grupos, cada um contendo oito animais, de acordo com a medicação adminis-

* Trabalho realizado no Laboratório de Cirurgia Experimental da Faculdade de Ciências Médicas - Universidade do Estado do Rio de Janeiro - UERJ - Rio de Janeiro (RJ), Brasil.

1. Doutor, Professor Adjunto da Disciplina de Ortopedia e Traumatologia da Faculdade de Ciências Médicas da Universidade do Estado do Rio de Janeiro - UERJ - Rio de Janeiro (RJ), Brasil.

2. Mestre, Professor Assistente da Disciplina de Ortopedia e Traumatologia da Faculdade de Ciências Médicas da Universidade do Estado do Rio de Janeiro - UERJ - Rio de Janeiro (RJ), Brasil.

3. Doutor, Professor Adjunto do Instituto de Biologia Roberto Alcântara Gomes - Universidade do Estado do Rio de Janeiro UERJ - Rio de Janeiro (RJ), Brasil.

4. Professor Titular da Disciplina de Ortopedia e Traumatologia da Faculdade de Ciências Médicas - Universidade do Estado do Rio de Janeiro - UERJ - Rio de Janeiro (RJ), Brasil.

Endereço para correspondência: Av. Érico Veríssimo, 901, sala 201, Barra da Tijuca - 22631-004 - Rio de Janeiro, RJ. Tel.: (21) 24958430. E-mail: lisztpalmeira@yahoo.com.br

Recebido em 5/6/07. Aprovado para publicação em 10/12/07. Copyright RBO2007 trada diariamente por 14 dias: $\mathrm{HC}(25 \mathrm{mg}), \mathrm{HC}$ $(25 \mathrm{mg})+\mathrm{SV}, \mathrm{HC}(1 \mathrm{mg}), \mathrm{HC}(\mathbf{1 m g})+\mathrm{SV}$ e soro fisiológico (SF). Foram realizadas dosagens séricas de triglicerídeos (TRI), colesterol total (COL), aspartato aminotransferase (AST) e alanina aminotransferase (ALT), antes e depois do tratamento. A AMOA da cabeça femoral foi determinada por morfometria. Resultados: Os grupos foram semelhantes no início do experimento, com relação aos valores séricos de TRI, COL, AST e ALT. Os animais em que foi administrado HC apresentaram maior AMOA, quando comparados com os que receberam $\mathrm{SF}(\mathrm{p}<\mathbf{0 , 0 0 0 1})$, e elevação estatisticamente significativa nas dosagens séricas de TRI e COL. A AMOA foi maior nos grupos HC do que nos HC $+\mathrm{SV}(\mathrm{p}<\mathbf{0 , 0 0 0 1})$. No grupo HC (1mg) + SV, não houve diferença significativa na AMOA, quando comparado com o SF $(p=0,5047)$, nem nos valores séricos de TRI $(p=0,1907)$ e COL $(p=0,4480)$, depois do tratamento. Nos animais que receberam $\mathrm{HC}(25 \mathrm{mg})+\mathrm{SV}$, houve diferença significativa na AMOA, quando comparados com os do grupo SF ( $<<0,0001)$, e nas medidas séricas de TRI $(p=0,0044)$ e COL $(p=0,0025)$, depois do tratamento. Todos os grupos apresentaram elevação dos níveis séricos de AST e ALT. O uso de HC se relacionou à maior área ocupada por adipócitos na medula óssea da cabeça femoral, diretamente proporcional à dose administrada. Esse efeito se reduziu com o uso de sinvastatina. Conclusão: $O$ efeito da sin- 
vastatina na redução da intensidade do aumento do conteúdo lipídico da medula óssea induzida pelo uso de HC foi significativo, sendo mais consistente no grupo tratado com baixas doses de $\mathrm{HC}$.

\author{
Descritores - Corticosteróides; Sinvastatina; Inibidores de \\ hidroximetilglutaril-CoA redutases; Medula \\ óssea/efeito de drogas
}

\section{ABSTRACT}

Objectives: To evaluate the effects of using simvastatin (SV) on the medullary area occupied by adipocytes (MAOA) in the femoral head of rats with lipidic hypertrophy induced by hydrocortisone (HC). Methods: 40 male Wistar rats (Rattus norvegicus albinos) were distributed into five groups, each group with eight animals, according to the medication given daily during 15 days: HC (25 mg), $H C(25 m g)+S B, H C(1 m g), H C(1 m g)+S V$, and saline solution (SF). Serum triglyceride (TRI), total cholesterol (COL), aspartate aminotransferase (AST), and alanine aminotransferase (ALT) tests were performed before and after treatment. Femoral head MAOA was determined by morphometry. Results: the groups had similar results of serum values of TRI, COL, AST, and ALT in the beginning of the experiment. The animals that received $H C$ had greater MAOA when compared to those receiving $S F(p<0.0001)$, with statistically significant increases in the serum TRI and COL readings. MAOA was greater in the groups receiving $H C$ than in the groups receiving $J C$ $+S V(p<0.0001)$. In the group that received HC $(1$ $m g)+S V$, there was no significant difference in $M A O A$, when compared to the group that received $S F$ $(p=0.5047)$, nor in the serum values of TRI ( $p=$ $0.1907)$ and $\operatorname{COL}(p=0.4480)$, after the treatment. In the animals that received $H C(25 \mathrm{mg})+S V$, there was a significant difference in $M A O A$, when compared to the group that received $S F(p<0.0001)$, and in the serum readings of TRI $(p=0.0044)$ and $\operatorname{COL}(p=$ $0.0025)$, after the treatment. All groups presented increased serum levels of AST and ALT. The use of HC was related to a larger area occupied by adipocytes in the medulla of the femoral head, which was directly proportional to the dose given. This effect was reduced with the use of simvastatin. Conclusion: The use of simvastatin to reduce the bone medulla lipidic content induced by HC was significant, and more consistent in the group treated with low HC doses.

\section{Keywords - Adrenal cortex hormones; Simvastatin; Hydroxymethylglutaryl-CoA reductase inhibitors; Bone marrow/drug effects}

\section{INTRODUÇÃO}

A osteonecrose (ON) é entidade nosológica específica, mas resultado final de uma série de alterações que produzem a diminuição do fluxo sanguíneo intraósseo e acarretam a morte celular no tecido ósseo, com subseqüente destruição de sua arquitetura estrutural. Diferentes termos têm sido utilizados como sinônimos de ON, tais como necrose avascular, necrose isquêmica e necrose asséptica do osso.

Seu reconhecimento foi atribuído a James Russell que a caracterizou, em 1794, como entidade de origem infecciosa ${ }^{(1)}$. Em 1888, o termo necrose asséptica do osso foi dado ao que hoje se conhece como $\mathrm{ON}^{(2)}$.

A associação entre o desenvolvimento de ON e o uso dos glicocorticóides foi descrita em $1957^{(3)}$. Cerca de $35 \%$ dos casos de ON são associados ao uso desses medicamentos $^{(4-6)}$.

Embora ainda não esteja esclarecido o mecanismo pelo qual os glicocorticóides atuam na medula óssea, há indícios de que ele esteja relacionado com o estimulo à diferenciação das células mesenquimais pluripotenciais em adipócitos, suprimindo a diferenciação em osteoblastos e produzindo acúmulo de gordura ${ }^{(7-13)}$.

Em modelos experimentais com animais e em cultura de células mesenquimais submetidos à ação de glicocorticóides, o uso de estatina previne o aparecimento de alterações histológicas características da diferenciação lipídica da medula óssea ${ }^{(7,14)}$. Em seres humanos, a associação entre o uso de estatina e a diminuição do risco de desenvolvimento de ON induzida pelo uso de glicocorticóides foi observada em pacientes que iniciaram tratamento com altas doses de corticosteróides na vigência do uso desse fármaco ${ }^{(15)}$. 
Pelo exposto, a administração prévia de estatinas em animais que receberão glicocorticóides poderia ser vista como alternativa terapêutica eficaz na prevenção de alterações da medula óssea potencialmente relacionadas ao desenvolvimento de ON. Julgamos especialmente relevante testar essa hipótese nas fases iniciais do uso de glicocorticóides.

\section{MÉTODOS}

Os experimentos foram realizados no Laboratório de Cirurgia Experimental da Faculdade de Ciências Médicas da Universidade do Estado do Rio de Janeiro, com animais provenientes do biotério do próprio laboratório. O projeto de pesquisa foi aprovado pela Comissão de Ética em Pesquisa com Animais do laboratório. Foram utilizados 40 ratos machos (Rattus norvegicus albinus) da linhagem Wistar, com idade entre 60 e 70 dias e peso corporal variando entre $226 \mathrm{~g}$ e $292 \mathrm{~g}$ (média de 262g). Os animais foram aleatoriamente distribuídos em cinco grupos (A, B, C, D e E), com oito animais em cada um. Após 12 horas de jejum noturno, cada animal foi submetido à coleta de $0,8 \mathrm{ml}$ de sangue destinado à análise laboratorial de triglicerídeos (TRI), colesterol total (COL), aspartato aminotransferase (AST) e alanina aminotransferase (ALT), no Laboratório Central de Patologia Clínica do Hospital Universitário Pedro Ernesto - UERJ. A coleta do sangue foi realizada por dissecção de veia jugular, sob anestesia com tiopental sódico a $5 \mathrm{mg}$ para cada $100 \mathrm{~g}$ de peso, diluído em água destilada e inoculado por via intraperitoneal.

O glicocorticóide escolhido - succinato sódico de hidrocortisona (HC) - foi administrado por via subcutânea, diluído em solução salina fisiológica pelo período de 14 dias. Os animais dos grupos A e B receberam a dose diária de $25 \mathrm{mg}$ de $\mathrm{HC}$ e os dos grupos $\mathrm{C}$ e $\mathrm{D}$, dose diária de $1 \mathrm{mg}$ de HC. O grupo $\mathrm{E}$ (controle) recebeu soro fisiológico (SF), sem HC.

A dose de sinvastatina, administrada foi de $5 \mathrm{mg}$ por dia para cada animal. A administração diária de sinvastatina teve início 14 dias antes do começo do tratamento com hidrocortisona, sendo mantida durante todo o período de corticoterapia. A administração de sinvastatina (SV) foi feita nos animais dos grupos B e D.
Os animais dos grupos A, C e E receberam, no mesmo período, administração de água destilada, sem sinvastatina.

Após o término do período de administração dos medicamentos, todos os animais foram submetidos a um período noturno de 12 horas de jejum, sendo novamente coletado $0,8 \mathrm{ml}$ de sangue para análise laboratorial (TRI, COL, AST e ALT). Após a coleta de sangue, os animais foram sacrificados com dose letal de tiopental sódico, por via intraperitoneal.

Morfometria - As cabeças femorais foram divididas ao meio, no plano coronal, tendo como referência a inserção do ligamento redondo. As peças foram imediatamente fixadas em solução de glutaraldeído a 2,5\%, em tampão cacodilato de sódio $0,1 \mathrm{M}, \mathrm{pH} 7,2$, por duas horas, e lavadas em três banhos, de 10 minutos cada, no mesmo tampão. A seguir, foram descalcificadas, por sete dias, em ácido etilediaminotetracético (EDTA). A pós-fixação foi feita com tetróxido de ósmio $\left(\mathrm{OsO}_{4}\right)$, em tampão cacodilato de sódio $0,1 \mathrm{M}, \mathrm{pH} 7,2$, por uma hora, seguida de banho no mesmo tampão. As peças foram desidratadas em etanol $(30 \%, 50 \%, 70 \%$ e $100 \%$ ), tratadas com solução de óxido de propilenoepon em partes iguais e impregnados em epon durante seis horas em geladeira. A seguir, foram incluídas em epon por 48 horas a $60^{\circ} \mathrm{C}$, para polimerização. Cortes da região central da cabeça femoral, realizados no plano coronal e com espessura de $1 \mu \mathrm{m}$ foram obtidos em ultramicrótomo e corados em azul de toluidina a $0,5 \%$. Foram obtidos, de cada cabeça femoral, dois cortes de $1 \mu \mathrm{m}$ e analisados três campos por corte, perfazendo 12 campos por animal, para obtenção da média de ocupação percentual, por adipócitos, da área total da medula. As imagens das lâminas foram capturadas em um microscópio Olympus $B X-40^{\circledR}$, equipado com câmera digital Optronics $^{\circledR}$, armazenadas em formato TIFF (tagged image file format) e analisadas pelo programa Image Pro Plus ${ }^{\circledR}$ (Media Cybernetics). Os adipócitos foram facilmente identificados pela forma arredondada e pela tênue coloração característica da gordura verificada após a fixação pelo tetróxido de ósmio. A área relativa ocupada por adipócitos foi calculada no tecido hematopoético da medula óssea. 


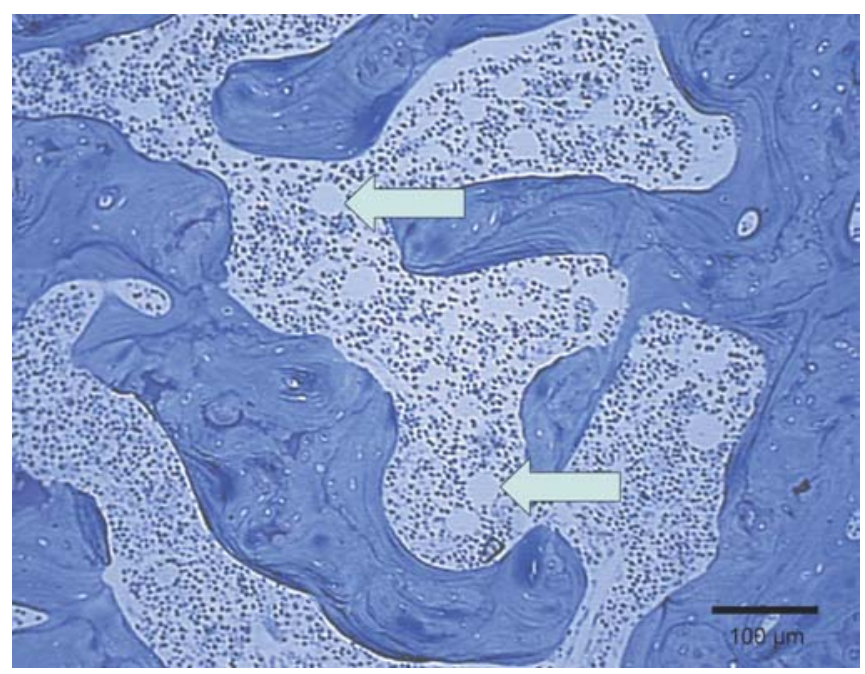

Figura 1 - Fotomicrografia da medula óssea da cabeça femoral de rato do grupo SF. Observa-se a presença de células adiposas (setas) na medula hematopoética. Azul de toluidina 0,5\%. Barra de calibração $100 \mu \mathrm{m}$.

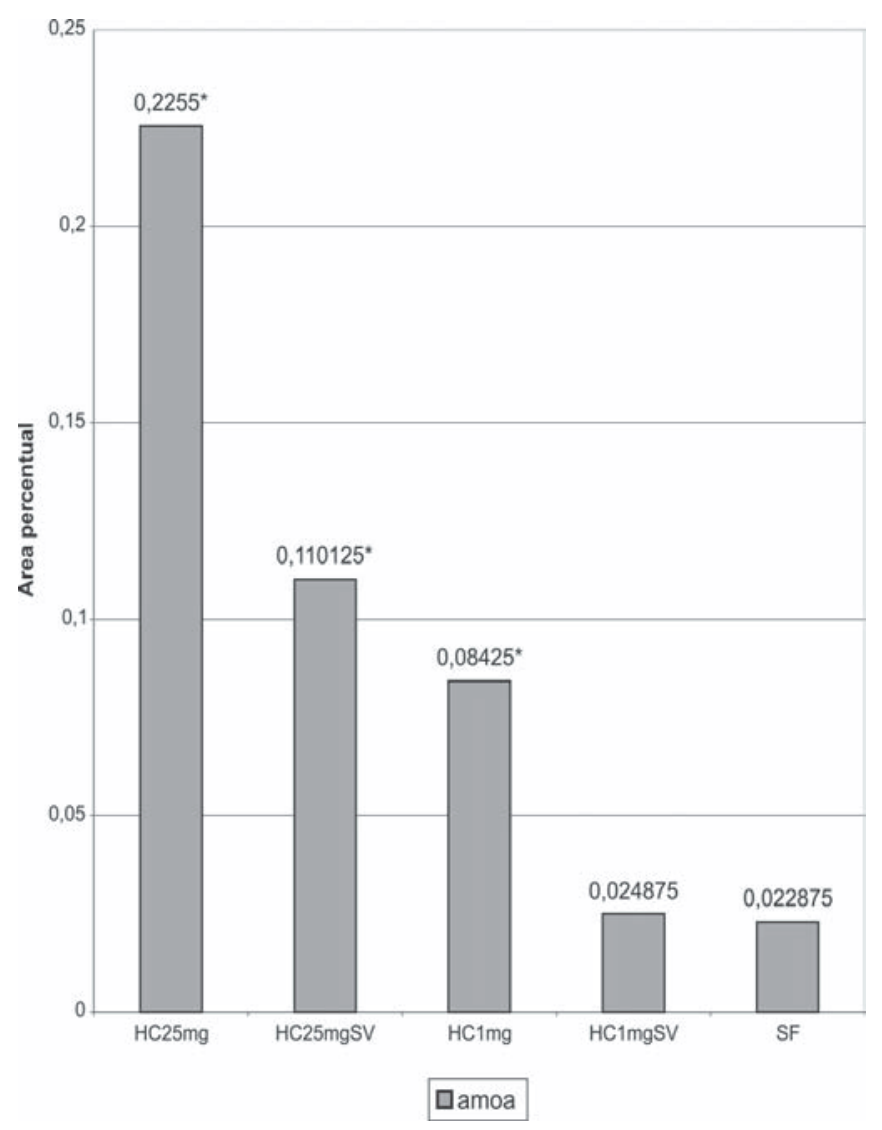

Figura 3 - Área da medula óssea ocupada por adipócitos (amoa). * diferença estatisticamente significativa em relação ao grupo controle $(p<0,0001)$.

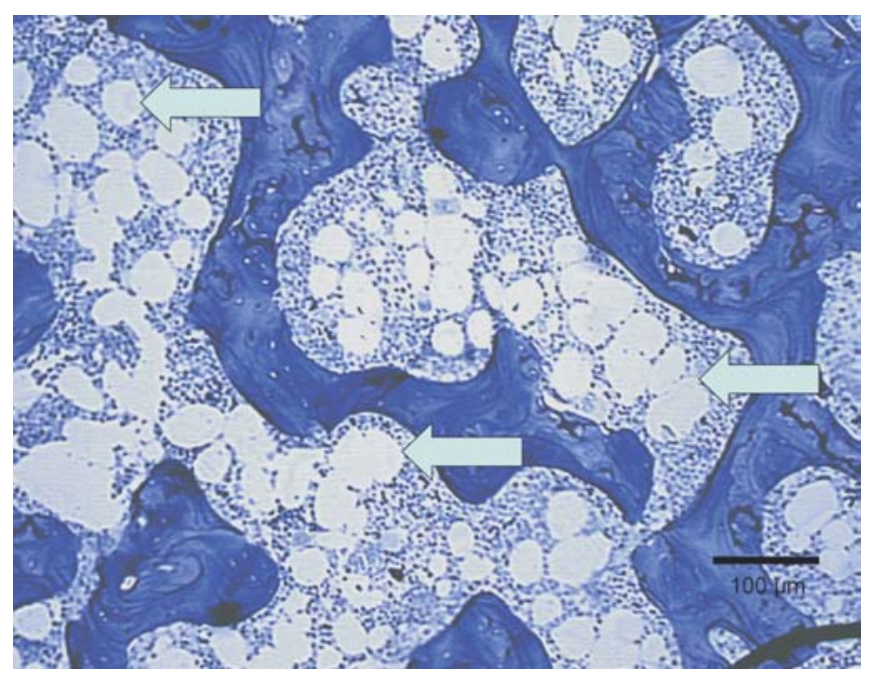

Figura 2 - Fotomicrografia da medula óssea da cabeça femoral de rato do grupo $\mathrm{A}$ ( $\mathrm{HC} 25 \mathrm{mg})$. Observa-se a presença de número aumentado de células adiposas (setas) na medula hematopoética. Azul de toluidina 0,5\%. Barra de calibração $100 \mu \mathrm{m}$.

Análise estatística - Para verificar a existência de diferença entre as médias dos valores de peso corporal, dosagens laboratoriais e área da medula óssea ocupada por adipócitos, foi utilizado o teste $t$. Para comparação entre as médias obtidas nas dosagens laboratoriais, antes e depois do tratamento, foi aplicado o teste $t$ pareado.

\section{RESULTADOS}

A administração de corticosteróides pelo período de 14 dias foi relacionada a elevado conteúdo lipídico na medula óssea da cabeça femoral (figuras 1 e 2). A figura 3 ilustra as médias da área da medula óssea ocupada por adipócitos (AMOA) em cada um dos grupos de animais. Foi observada diferença significativa na AMOA nos ratos dos grupos A, B e C, quando comparados com os do grupo controle (tabela 1). O grau de hipertrofia lipídica na medula óssea da cabeça femoral foi maior após o tratamento com a dose de $25 \mathrm{mg}$ / $\mathrm{kg} /$ dia do que com a dose de $1 \mathrm{mg} / \mathrm{kg} /$ dia, evidenciado pela diferença na AMOA entre os grupos A e C.

$\mathrm{O}$ efeito da sinvastatina na redução do desenvolvimento da diferenciação lipídica medular causada pelos glicocorticóides foi verificado pela diferença significativa na AMOA entre os grupos A e B e entre os 
TABELA 1

Área da medula óssea ocupada por adipócitos. Comparação com o grupo controle

\begin{tabular}{llllll}
\hline \multicolumn{1}{c}{ Grupos } & N & Média & $\begin{array}{l}\text { Desvio } \\
\text { padrão }\end{array}$ & 95\% IC & \\
\hline A (HC25mg) & 8 & 0,2255 & 0,028 & $(0,2020-0,2489)$ & $\mathrm{p}<0,0001$ \\
B (HC25mgSV) & 8 & 0,1101 & 0,01893 & $(0,0942-0,1259)$ & $\mathrm{p}<0,0001$ \\
C (HC1mg) & 8 & 0,0842 & 0,0084 & $(0,0771-0,0913)$ & $\mathrm{p}<0,0001$ \\
D (HC1mgSV) & 8 & 0,0248 & 0,0067 & $(0,0192-0,0304)$ & $\mathrm{p}>0,5$ \\
$\mathrm{E}$ (Controle) & 8 & 0,0228 & 0,0048 & $(0,0188-0,0269)$ & \\
\hline
\end{tabular}

Fonte: Laboratório de Cirurgia Experimental - FCM-UERJ.

Legenda: N: Número de animais por grupo; IC: Intervalo de Confiança; HC: hidrocor tisona; SV: sinvastatina.

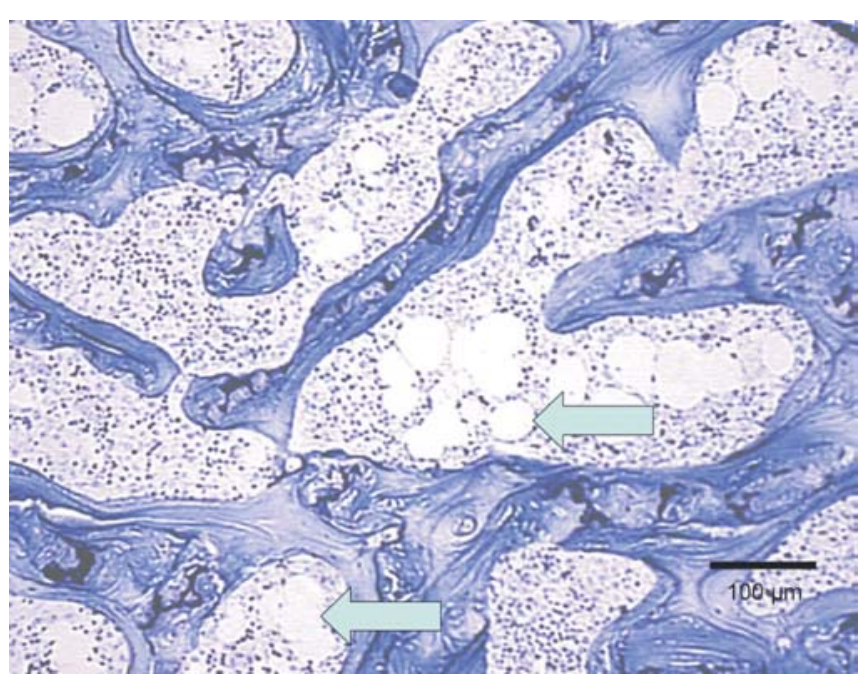

Figura 4 - Fotomicrografia da medula óssea da cabeça femoral de rato do grupo $\mathrm{C}$ (HC1mg). Observa-se discreto aumento do número de células adiposas (setas) na medula hematopoética. Azul de toluidina 0,5\%. Barra de calibração $100 \mu \mathrm{m}$.

grupos $\mathrm{C}$ e D. Em ambos os casos, a AMOA foi menor no grupo que recebeu sinvastatina. No grupo tratado com sinvastatina associada a hidrocortisona em dose baixa $(1 \mathrm{mg} / \mathrm{kg} / \mathrm{dia})$, não houve diferença significativa na AMOA, quando comparado com o grupo que recebeu SF sem hidrocortisona (figura 4).

Foram verificadas diferenças entre as dosagens laboratoriais realizadas no início e ao final do período de administração dos medicamentos em todos os grupos de animais, com tendência a aumento, na segunda amostra, nos valores séricos de TRI, COL, AST e ALT. Foi verificada diferença significativa entre as dosagens séricas de TRI, COL, AST e ASL nas amostras obtidas
TABELA 2

Resultados laboratoriais grupo A (HC25mg)

\begin{tabular}{lcccc}
\hline \multicolumn{1}{c}{ Medidas } & Média & $\begin{array}{c}\text { Desvio } \\
\text { padrão }\end{array}$ & 95\% IC & \\
\hline TRI mg/dl (antes) & 50 & 11,18 & $(40,64-59,35)$ & \\
TRI mg/dl (depois) & 73,75 & 6,9 & $(67,97-79,52)$ & $\mathrm{p}=0,0003$ \\
\hline COL mg/dl (antes) & 67,12 & 5,61 & $(62,42-71,82)$ & \\
COL mg/dl (depois) & 89,87 & 10,5 & $(81,09-98,65)$ & $p=0,0025$ \\
\hline AST UI (antes) & 169,62 & 20,09 & $(152,82-186,42)$ & \\
AST UI (depois) & 200,75 & 24,34 & $(180,4-221,09)$ & $p=0,0011$ \\
\hline ALT UI (antes) & 69 & 7,74 & $(62,52-75,47)$ & \\
ALT UI (depois) & 77,37 & 11,18 & $(68,02-86,72)$ & $p=0,0031$ \\
\hline
\end{tabular}

Fonte: Laboratório de Cirurgia Experimental - FCM-UERJ.

Legenda: N: Número de animais por grupo; IC: Intervalo de Confiança; HC: hidrocortisona; TRI: triglicerídeos; COL: colesterol; AST: aspartato aminotransferase; ALT: alanina aminotransferase.

TABELA 3

Resultados laboratoriais grupo B (HC25mgSV)

\begin{tabular}{lcccc}
\hline \multicolumn{1}{c}{ Medidas } & Média & $\begin{array}{c}\text { Desvio } \\
\text { padrão }\end{array}$ & 95\% IC & \\
\hline TRI mg/dl (antes) & 56,75 & 16,96 & $(42,56-70,93)$ & \\
TRI mg/dl (depois) & 69,25 & 10,7 & $(60,3-78,19)$ & $\mathrm{p}=0,0044$ \\
\hline COL mg/dl (antes) & 68,75 & 8,48 & $(61,65-75,84)$ & \\
COL mg/dl (depois) & 80,37 & 9,22 & $(72,66-88,08)$ & $\mathrm{p}=0,0025$ \\
\hline AST UI (antes) & 163,62 & 17,01 & $(149,40-177,84)$ & \\
AST UI (depois) & 218,25 & 25,47 & $(196,95-239,54)$ & $\mathrm{p}=0,0014$ \\
\hline ALT UI (antes) & 69 & 8,07 & $(62,25-75,74)$ & \\
ALT UI (depois) & 85,12 & 8,74 & $(77,81-92,43)$ & $\mathrm{p}=0,0036$
\end{tabular}

Fonte: Laboratório de Cirurgia Experimental - FCM-UERJ.

Legenda: N: Número de animais por grupo; IC: Intervalo de Confiança; HC: hidrocortisona; SV: sinvastatina; TRI: triglicerídeos; COL: colesterol; AST: aspartato aminotransferase; ALT: alanina aminotransferase.

antes e depois da administração dos medicamentos nos grupos A (tabela 2) e B (tabela 3), que receberam HC em altas doses. Na comparação entre esses dois grupos, a utilização de sinvastatina não foi relacionada a aumento significativo das aminotransferases AST e ALT.

Entre os dois grupos que receberam a dose de $1 \mathrm{mg} /$ $\mathrm{kg} /$ dia de HC, houve diferença nos resultados obtidos com as dosagens laboratoriais. No grupo que recebeu somente $\mathrm{HC}$, houve diferença significativa entre os valores séricos de antes e depois, para as dosagens de TRI e AST; as dosagens de COL e ALT, nesse grupo, não 
TABELA 4

Resultados laboratoriais grupo C (HC1mg)

\begin{tabular}{lcrccc}
\hline \multicolumn{1}{c}{ Medidas } & Média & $\begin{array}{r}\text { Desvio } \\
\text { padrão }\end{array}$ & $95 \%$ IC & \\
\hline TRI mg/dl (antes) & 53,25 & 5,82 & $(48,38-58,11)$ & \\
TRI mg/dl (depois) & 61,62 & 6,27 & $(56,37-66,87)$ & $\mathrm{p}=0,0012$ \\
\hline COL mg/dl (antes) & 69,75 & 6,92 & $(63,96-75,53)$ & \\
COL mg/dl (depois) & 78,87 & 8,57 & $(71,70-86,04)$ & $\mathrm{p}=0,0554$ \\
\hline AST UI (antes) & 174,87 & 16,37 & $(161,18-188,56)$ & \\
AST UI (depois) & 194,12 & 8,93 & $(186,65-201,59)$ & $\mathrm{p}=0,0035$ \\
\hline ALT UI (antes) & 67,87 & 13,08 & $(56,93-78,81)$ & \\
ALT UI (depois) & 74,5 & 9,24 & $(66,77-82,22)$ & $\mathrm{p}=0,0605$ \\
\hline
\end{tabular}

Fonte: Laboratório de Cirurgia Experimental - FCM-UERJ.

Legenda: N: Número de animais por grupo; IC: Intervalo de Confiança; HC: hidrocortisona; TRI: triglicerídeos; COL: colesterol; AST: aspartato aminotransferase; ALT: alanina aminotransferase.

apresentaram diferença significativa entre as duas coletas (tabela 4). No grupo D, que recebeu $1 \mathrm{mg}$ de $\mathrm{HC}$ associada a SV, foi verificada nítida diferença no perfil lipídico laboratorial. As diferenças entre as médias das dosagens séricas de TRI e COL, realizadas antes e depois, nesse grupo, não foram significativas (tabela 5). Foi observada, nesse grupo, elevação significativa dos valores das transaminases entre a coleta inicial e a final. O perfil dos resultados laboratoriais do grupo D foi similar ao obtido no grupo controle, em que também foi verificada elevação de AST e ALT, com manutenção dos níveis séricos de TRI e COL, entre as duas coletas realizadas (tabela 6 ).

\section{DISCUSSÃO}

Neste estudo, houve aumento significativo na área ocupada por adipócitos da medula óssea da cabeça femoral nos animais que foram submetidos à administração de hidrocortisona por 14 dias consecutivos, quando comparados com os do grupo de animais não tratados com HC. O aumento do conteúdo lipídico da medula óssea foi observado após a administração de glicocorticóides em diversos estudos experimentais in vitro e in vivo ${ }^{(7,14,16-19)}$. Em cultura de células mesenquimais da medula óssea, a administração de glicocorticóides diminui a expressão genética do fator de transcrição celular Cbfa1/Runx2 e a atividade da os-
TABELA 5

Resultados laboratoriais grupo D (HC1mgSV)

\begin{tabular}{llllll}
\hline \multicolumn{1}{c}{ Medidas } & Média & $\begin{array}{c}\text { Desvio } \\
\text { padrão }\end{array}$ & $95 \%$ IC & \\
\hline TRI mg/dl (antes) & 54 & 7,55 & $(47,68-50,31)$ & \\
TRI mg/dl (depois) & 57,37 & 5,57 & $(52,71-62,03)$ & $\mathrm{p}=0,1907$ \\
\hline COL mg/dl (antes) & 71,25 & 9,09 & $(63,64-78,85)$ & \\
COL mg/dl (depois) & 73 & 8,21 & $(66,13-79,86)$ & $\mathrm{p}=0,4480$ \\
\hline AST UI (antes) & 166,37 & 21,3 & $(148,56-184,18)$ & \\
AST UI (depois) & 214,5 & 19,07 & $(198,54-230,45)$ & $\mathrm{p}=0,0046$ \\
\hline ALT UI (antes) & 71,25 & 7,49 & $(64,98-77,51)$ & \\
ALT UI (depois) & 87,12 & 8,65 & $(79,88-94,36)$ & $\mathrm{p}=0,0001$
\end{tabular}

Fonte: Laboratório de Cirurgia Experimental - FCM-UERJ.

Legenda: N: Número de animais por grupo; IC: Intervalo de Confiança; HC: hidrocortisona; SV: sinvastatina; TRI: triglicerídeos; COL: colesterol; AST: aspartato aminotransferase; ALT: alanina aminotransferase.

TABELA 6

Resultados laboratoriais grupo $\mathrm{E}$ (controle)

\begin{tabular}{|c|c|c|c|c|}
\hline Medidas & Média & $\begin{array}{l}\text { Desvio } \\
\text { padrão }\end{array}$ & $95 \%$ IC & \\
\hline $\mathrm{TRI} \mathrm{mg} / \mathrm{dl}$ (antes) & 57,25 & 10,22 & $(48,7-65,79)$ & \\
\hline TRI mg/dl (depois) & 57 & 9,48 & $(49,06-64,93)$ & $p=0,9330$ \\
\hline $\mathrm{COL} \mathrm{mg/dl} \mathrm{(antes)}$ & 70,5 & 11,97 & $(60,48-80,51)$ & \\
\hline COL mg/dl (depois) & 68,87 & 7,69 & $(62,43-75,31)$ & $p=0,6954$ \\
\hline AST UI (antes) & 173,75 & 17,88 & $(158,79-188,70)$ & \\
\hline AST UI (depois) & 193,12 & 14,38 & $(181,09-205,15)$ & $p=0,0020$ \\
\hline ALT UI (antes) & 71,125 & 11,45 & $(61,54-80,70)$ & \\
\hline ALT UI (depois) & 79,12 & 8,18 & $(72,28-85,96)$ & $p=0,0036$ \\
\hline
\end{tabular}

Fonte: Laboratório de Cirurgia Experimental - FCM-UERJ.

Legenda: N: Número de animais por grupo; IC: Intervalo de Confiança; TRI: triglicerídeos; COL: colesterol; AST: aspartato aminotransferase; ALT: alanina aminotransferase.

teocalcina, ao mesmo tempo em que eleva a expressão genética do fator de transcrição celular PPARgamma2, indicando o aumento da adipogênese e a diminuição da osteogênese pelas células mesenquimais in vitro ${ }^{(20)}$.

De forma semelhante ao observado em outros estudos, verificamos que ocorrem alterações ósseas características de estímulo de diferenciação lipídica nos animais submetidos ao uso de corticosteróides, nas duas primeiras semanas após o início da administração dos medicamentos ${ }^{(14,21-22)}$. Autores demonstraram a ocorrência de proliferação de adipócitos na medula 
óssea em até 48 horas após a administração de glicocorticóides, com aumento progressivo no número e tamanho das células adiposas nas primeiras quatro semanas ${ }^{(7,14,23-24)}$. A ON é considerada, portanto, consequiência da administração de glicocorticóides e o aumento do conteúdo lipídico da medula óssea poderia ser considerado uma das manifestações histológicas iniciais.

A partir dos resultados obtidos com a avaliação morfométrica da medula óssea da cabeça femoral de ratos tratados com glicocorticóides em dose alta e baixa, pudemos verificar que, efetivamente, existe relação entre a intensidade do efeito verificado na medula óssea e a dose utilizada. Nas duas doses utilizadas, os efeitos verificados após a administração de glicocorticóides nos animais foram no sentido de aumento de número e volume das células adiposas na medula óssea, quando comparados com os do grupo controle, que não fez uso dos glicocorticóides. A intensidade do efeito foi significativamente maior no grupo no qual foi administrada a dose mais elevada, quando comparado com o grupo que recebeu a dose mais baixa. Outros autores também verificaram que o grau de hipertrofia lipídica da medula óssea e a prevalência de osteonecrose estão relacionados ao uso de doses mais altas de glicocorticóides ${ }^{(25-28)}$. O número de células com vesículas de triglicerídeos e a expressão de genes específicos de células lipídicas, em cultura de células pluripotenciais tratadas com dexametasona, são maiores quando são utilizadas doses mais elevadas de glicocorticóides $^{(7,14)}$.

Alterações no metabolismo lipídico foram demonstradas em pacientes com osteonecrose e em animais submetidos a tratamento com glicocorticóides ${ }^{(14,16,19}$, ${ }^{21,29)}$. No nosso estudo, observamos alterações no metabolismo lipídico nos animais tratados com glicocorticóides, caracterizadas pela elevação dos níveis séricos de colesterol total e triglicerídeos, quando comparadas as mensurações realizadas antes e depois do tratamento. Em células de linhagem pluripotencial, capazes de se diferenciar tanto em osteoblastos quanto em adipócitos, a administração de estatina foi responsável por aumento na expressão do fator de transcrição celular de osteoblastos (Cbfa1/Runx2) e pela dimi- nuição na expressão do fator de transcrição celular de adipócitos (PPARgamma2) ${ }^{(30)}$. A estatina pode, portanto, desviar células osteoprogenitoras da medula óssea do caminho da diferenciação lipídica para a diferenciação osteoblástica, pelo aumento da expressão genética osteoblástica e pela diminuição da adipogênese.

Neste estudo, o tratamento com a sinvastatina teve início 14 dias antes da administração de glicocorticóides, com o objetivo de potencializar o seu efeito, que parece estar relacionado com um período mínimo de indução $^{(31-33)}$. Observamos que o período de 14 dias de indução com sinvastatina, antes da administração do glicocorticóide, foi suficiente para apresentar os efeitos preventivos, evidenciados no nosso estudo, pela diferença significativa na AMOA entre os grupos tratados com corticosteróides e os grupos que fizeram uso de glicocorticóide e sinvastatina.

Os efeitos colaterais mais importantes dos inibidores da HMG-CoA redutase são a toxicidade hepática e muscular ${ }^{(34)}$. Embora nas doses comumente empregadas as estatinas não provoquem lesão hepática significativa $^{(35)}$, doses muito elevadas estão associadas ao desenvolvimento de necrose hepatocelular em animais de laboratório ${ }^{(36-38)}$. A manifestação hepática mais comum relacionada ao uso de estatinas é a elevação assintomática das transaminases ${ }^{(39-40)}$. No nosso estudo, verificamos elevação do nível sérico das aminotransferases hepáticas entre as dosagens realizadas antes e depois da administração dos medicamentos, em todos os grupos, inclusive no grupo controle. A elevação foi significativamente maior nos animais dos grupos nos quais foi administrada a sinvastatina, em comparação com os animais dos outros grupos. Em recente revisão da literatura, Chalasani ${ }^{(41)}$ observou que essa elevação também ocorreu com freqüência similar nos pacientes tratados com placebo nos estudos clínicos. Esses achados levantam a possibilidade de que pacientes com hiperlipidemia possam ter flutuações espontâneas nas transaminases, tenham recebido ou não estatinas, e podem explicar também a elevação assintomática das aminotransferases que ocorre com agentes redutores de lipídios que não atuam na síntese hepática de colesterol $^{(38)}$. O mecanismo pelo qual as estatinas cau- 
sam a elevação dessas enzimas não é completamente esclarecido. Alguns autores acreditam que o aumento das aminotransferases hepáticas pode representar um efeito farmacodinâmico da redução lipídica, não tendo relação direta com a estatina ${ }^{(42)}$.

\section{CONCLUSÃO}

Os resultados obtidos neste estudo indicam que a administração de HC produziu aumento significativo na área ocupada por adipócitos na medula óssea da cabeça femoral, havendo relação direta entre a intensidade do efeito e a dose de HC aplicada. O efeito da $\mathrm{HC}$, nas duas doses utilizadas, foi reduzido de forma significativa com o uso de sinvastatina.

\section{REFERÊNCIAS}

1. The Classic. An essay on necrosis. Section 1. General remarks, and description of appearances. Clin Orthop Relat Res. 1978; (130):5-7.

2. Springfield DS, Enneking WJ. Surgery for aseptic necrosis of the femoral head. Clin Orthop Relat Res. 1978;(130):175-85.

3. Kemper JW, Baggenstoss AH, Slocumb CH. The relationship of therapy with cortisone to the incidence of vascular lesions in rheumatoid arthritis. Ann Intern Med. 1957;46(5):831-51.

4. Jacobs B. Epidemiology of traumatic and nontraumatic osteonecrosis. Clin Orthop Relat Res. 1978;(130):51-67.

5. Jones JP Jr. Intravascular coagulation and osteonecrosis. Clin Orthop Relat Res. 1992;(277):41-53. Review.

6. Mankin HJ. Nontraumatic necrosis of bone (osteonecrosis). N Engl J Med. 1992;326(22):1473-9.

7. Cui Q, Wang GJ, Balian G. Steroid-induced adipogenesis in a pluripotential cell line from bone marrow. J Bone Joint Surg Am. 1997;79(7):1054-63.

8. Delany AM, Dong Y, Canalis E. Mechanisms of glucocorticoid action in bone cells. J Cell Biochem. 1994;56(3):295-302.

9. Dorheim MA, Sullivan M, Dandapani V, Wu X, Hudson J, Segarini PR, et al. Osteoblastic gene expression during adipogenesis in hematopoietic supporting murine bone marrow stromal cells. J Cell Physiol. 1993;154(2):317-28.

10. Gangji V, Hauzeur JP, Schoutens A, Hinsenkamp M, Appelboom T, Egrise D. Abnormalities in the replicative capacity of osteoblastic cells in the proximal femur of patients with osteonecrosis of the femoral head. J Rheumatol. 2003; 30(2):348-51.

11. Grigoriadis AE, Heersche JN, Aubin JE. Differentiation of muscle, fat, cartilage, and bone from progenitor cells present in a bone-derived clonal cell population: effect of dexamethasone. J Cell Biol. 1988;106(6):2139-51.
12. Hernigou P, Beaujean F, Lambotte JC. Decrease in the mesenchymal stem-cell pool in the proximal femur in corticosteroid-induced osteonecrosis. J Bone Joint Surg Br. 1999;81(2):349-55.

13. Liao JK. Isoprenoids as mediators of the biological effects of statins. J Clin Invest. 2002;110(3):285-8.

14. Wang GJ, Cui Q, Balian G. The Nicolas Andry award. The pathogenesis and prevention of steroid-induced osteonecrosis. Clin Orthop Relat Res. 2000;(370):295-310.

15. Pritchett JW. Statin therapy decreases the risk of osteonecrosis in patients receiving steroids. Clin Orthop Relat Res. 2001; (386):173-8.

16. Kawai K, Tamaki A, Hirohata K. Steroid-induced accumulation of lipid in the osteocytes of the rabbit femoral head. A histochemical and electron microscopic study. J Bone Joint Surg Am. 1985;67(5):755-63.

17. Locklin RM, Williamson MC, Beresford JN, Triffitt JT, Owen ME. In vitro effects of growth factors and dexamethasone on rat marrow stromal cells. Clin Orthop Relat Res. 1995;(313):2735.

18. Moran TJ. Cortisone-induced alterations in lipid metabolism. Morphologic and serologic observations in rabbits. Arch Pathol. 1962;73:300-12.

19. Wang GJ, Sweet DE, Reger SI, Thompson RC. Fat-cell changes as a mechanism of avascular necrosis of the femoral head in cortisone-treated rabbits. J Bone Joint Surg Am. 1977;59(6): 729-35.

20. Li X, Jin L, Cui Q, Wang GJ, Balian G. Steroid effects on osteogenesis through mesenchymal cell gene expression. Osteoporos Int. 2005;16(1):101-8.

21. Hungerford DS, Lennox DW. The importance of increased intraosseous pressure in the development of osteonecrosis of the femoral head: implications for treatment. Orthop Clin North Am. 1985;16(4):635-54.

22. Scófano Júnior AR, Silva MG, Souza I, Silva Filho NM, Mendes PHB, Elias N, Apfel MIR. Avaliação histológica das alterações da placa epifisária distal do fêmur após uso crônico de corticóide: estudo experimental. Rev Bras Ortop. 1999;34(8): 465-8.

23. Boumpas DT, Chrousos GP, Wilder RL, Cupps TR, Balow JE. Glucocorticoid therapy for immune-mediated diseases: basic and clinical correlates. Ann Intern Med. 1993;119(12):1198208.

24. Miyanishi K, Yamamoto T, Irisa T, Yamashita A, Jingushi S, Noguchi Y, Iwamoto Y. Bone marrow fat cell enlargement and a rise in intraosseous pressure in steroid-treated rabbits with osteonecrosis. Bone. 2002;30(1):185-90.

25. Bradbury G, Benjamin J, Thompson J, Klees E, Copeland J. Avascular necrosis of bone after cardiac transplantation. Prevalence and relationship to administration and dosage of steroids. J Bone Joint Surg Am. 1994;76(9):1385-8. 
26. Mont MA, Glueck CJ, Pacheco IH, Wang P, Hungerford DS, Petri M. Risk factors for osteonecrosis in systemic lupus erythematosus. J Rheumatol. 1997;24(4):654-62. Comment in: J Rheumatol. 1998;25(12):2477-8. J Rheumatol. 1998;25(1): 188.

27. Oinuma K, Harada Y, Nawata Y, Takabayashi K, Abe I, Kamikawa K, Moriya H. Osteonecrosis in patients with systemic lupus erythematosus develops very early after starting high dose corticosteroid treatment. Ann Rheum Dis. 2001; 60(12):1145-8.

28. Zonana-Nacach A, Barr SG, Magder LS, Petri M. Damage in systemic lupus erythematosus and its association with corticosteroids. Arthritis Rheum. 2000;43(8):1801-8.

29. Kawai K, Maruno H, Watanabe Y, Hirohata K. Fat necrosis of osteocytes as a causative factor in idiopathic osteonecrosis in heritable hyperlipemic rabbits. Clin Orthop Related Res. 1980; (153):273-82

30. Li X, Cui Q, Kao C, Wang GJ, Balian G. Lovastatin inhibits adipogenic and stimulates osteogenic differentiation by suppressing PPARgamma2 and increasing Cbfa1/Runx2 expression in bone marrow mesenchymal cell cultures. Bone. 2003;33(4):652-9.

31. Laufs U, Endres M, Stagliano N, Amin-Hanjani S, Chui DS, Yang SX, et al. Neuroprotection mediated by changes in the endothelial actin cytoskeleton. J Clin Invest. 2000;106(1):1524.

32. Riesen WF, Engler H, Risch M, Korte W, Noseda G. Short-term effects of atorvastatin on C-reactive protein. Eur Heart J. 2002; 23(10):794-9. Comment in: Eur Heart J. 2002;23(10):761-4. Eur Heart J. 2004;25(1):96.

33. Anbinder AL, Prado Fde A, Prado Mde A, Balducci I, da Rocha RF. The influence of ovariectomy, simvastatin and sodium alendronate on alveolar bone in rats. Braz Oral Res. 2007; 21(3):247-52.
34. Chitturi S, George J. Hepatotoxicity of commonly used drugs: nonsteroidal anti-inflammatory drugs, antihypertensives, antidiabetic agents, anticonvulsants, lipid-lowering agents, psychotropic drugs. Semin Liver Dis. 2002;22(2):169-83.

35. Kiortsis DN, Nikas S, Hatzidimou K, Tsianos E, Elisaf MS. Lipid-lowering drugs and serum liver enzymes: the effects of body weight and baseline enzyme levels. Fundam Clin Pharmacol. 2003;17(4):491-4.

36. Díaz-Zagoya JC, Asenjo-Barrón JC, Cárdenas-Vázquez R, Martínez F, Juárez-Oropeza MA. Comparative toxicity of high doses of vastatins currently used by clinicians, in CD-1 male mice fed with a hypercholesterolemic diet. Life Sci. 1999; 65(9):947-56.

37. Horsmans Y, Desager JP, Harvengt C. Biochemical changes and morphological alterations of the liver in guinea-pigs after administration of simvastatin (HMG CoA reductase-inhibitor). Pharmacol Toxicol. 1990;67(4):336-9.

38. MacDonald JS, Gerson RJ, Kornbrust DJ, Kloss MW, Prahalada S, Berry PH, et al. Preclinical evaluation of lovastatin. Am J Cardiol. 1988;62(15):16J-27J.

39. Parra JL, Reddy KR. Hepatotoxicity of hypolipidemic drugs. Clin Liver Dis. 2003;7(2):415-33.

40. Pasternak RC, Smith SC Jr, Bairey-Merz CN, Grundy SM, Cleeman JI, Lenfant C; American College of Cardiology; American Heart Association; National Heart, Lung and Blood Institute. ACC/AHA/NHLBI Clinical Advisory on the Use and Safety of Statins. Circulation. 2002;106(8):1024-8.

41. Chalasani N. Statins and hepatotoxicity: focus on patients with fatty liver. Hepatology. 2005;41(4):690-5.

42. Tolman KG. Defining patient risks from expanded preventive therapies. Am J Cardiol. 2000;85(12A):15E-9E. 